\title{
Ocorrência da tuberculose em profissionais da Saúde entre os anos 2014 e 2018 no Amapá
}

Occurrence of tuberculosis in health professionals and between the years 2014 and 2018 in Amapá

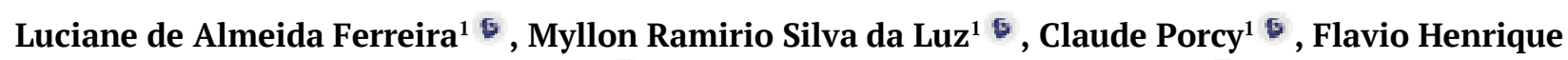 \\ Ferreira Barbosa $^{2} \sigma$, Rubens Alex de Oliveira Menezes ${ }^{3^{*}}$
}

${ }^{1}$ Faculdade Estácio de Macapá, Macapá, Amapá, Brasil. ${ }^{2}$ Departamento de Morfologia, Universidade Federal de Sergipe,
São Cristóvão, Sergipe, Brasil. ${ }^{3}$ Departamento de Ciências Biológicas e da Saúde, Universidade Federal do Amapá,
Macapá, Amapá, Brasil. *Autor para correspondência. E-mail: ra-menezes@hotmail.com/rubens.alex@unifap.br

Resumo: Introdução: Estudos indicam que profissionais de saúde estão altamente expostos ao risco de infecção por Mycobacterium tuberculosis, causador da tuberculose. No estado do Amapá ainda não há estudos direcionados especificamente para tuberculose em profissionais da área da saúde. Objetivo: Levantamento sobre ocorrência da tuberculose em profissionais da saúde do estado do Amapá entre os anos de 2014 e 2018. Material e métodos: Trata-se de um estudo descritivo de abordagem quantitativa no qual foram utilizados dados secundários obtidos a partir do Sistema de Informação de Agravos de Notificação (SINAN) por intermédio da Superintendência de Vigilância em Saúde (SVS) do Estado. As informações coletadas foram transferidas para uma planilha no aplicativo Microsoft Excel 2010 e em seguida para o programa Tabwin versão 4.1.5, onde foram realizados os cálculos e a tabulação dos resultados. Resultados e discussão: Foram diagnosticados 28 casos de tuberculose em profissionais da saúde entre 2014 e 2018 no Estado. O município do Macapá registrou o maior número de casos $(85,7 \%)$, a forma predominante foi a pulmonar (90\%), o sexo feminino (68\%) e a raça parda (68\%) foram mais atingidos e a faixa etária dos 20 aos 29 apresentou o maior número de casos. Conclusão: Ressalta-se a importância da completude nos dados fornecidos pelo SINAN e a importância do conhecimento da presença de tuberculose em profissionais da saúde no estado.

Palavras-chave: epidemiologia, Mycobacterium tuberculosis, profissionais da saúde, Região Amazônica, saúde pública, Tuberculose.

\begin{abstract}
Background: Studies indicate that health professionals are highly exposed to the risk of infection with Mycobacterium tuberculosis, which causes tuberculosis. In the state of Amapá, there are still no studies specifically targeting tuberculosis in healthcare professionals. Objective: Survey on the occurrence of tuberculosis in health professionals in the state of Amapá between the years 2014 and 2018. Material and methods: It is a descriptive study with a quantitative approach in which secondary data obtained from the Notifiable Diseases Information System (SINAN) through the State Health Surveillance Superintendence (SVS) were used. The collected information was transferred to a spreadsheet in the Microsoft Excel 2010 application and then to the Tabwin program version 4.1.5, where the results were calculated and tabulated. Results and discussion: 28 cases of tuberculosis in health professionals were diagnosed between 2014 and 2018 in the state. The municipality of Macapá recorded the highest number of cases (85.7\%), the predominant form was pulmonary (90\%), female (68\%) and brown race (68\%) were more affected and the age group from 20 to 29 presented the highest number of cases. Conclusion: The importance of completeness in the data provided by SINAN and the importance of knowledge of the presence of tuberculosis in health professionals in the state are emphasized.
\end{abstract}

Keywords: epidemiology, Mycobacterium tuberculosis, health personnel, Amazon region, public health, Tuberculosis.

\section{Introdução}

A tuberculose é uma doença infectocontagiosa transmitida por via aérea, causada pelo Mycobacterium tuberculosis, uma micobactéria pertencente ao gênero Mycobacterium, família Mycobacteriaceae, conhecido também por bacilo de Koch, denominação decorrente do nome da primeira pessoa a descrever a bactéria, Robert Koch, em 1882, na Alemanha (Campos, 2006).

Segundo a Organização Mundial da saúde a tuberculose continua sendo uma das principais causas de morte provocadas por um agente infeccioso no mundo. A estimativa foi de que em 2017 cerca 10 milhões de 
pessoas contraíram tuberculose no mundo e cerca 1,3 milhão de pessoas perderam a vida em decorrência da doença (WHO, 2018).

Os indicadores que revelam a incidência e a mortalidade por tuberculose no Brasil demonstram que a doença ainda ocorre com muita frequência no país. Em 2018 foram diagnosticados 72.788 casos novos de tuberculose de país, destes, 213 foram registrados no estado do Amapá. Os óbitos causados pela doença atingiram o número de 4.534 em 2017, 4 ocorreram no Amapá (Brasil, 2019).

De modo geral, os profissionais estão expostos aos riscos relacionados à sua profissão, direta ou indiretamente. Em profissões relacionadas à saúde há o risco biológico, que inclui a infecção por microrganismos oportunistas, os quais podem causar doenças que põem em risco a vida dos profissionais. Um exemplo é a tuberculose, cuja ocorrência em profissionais da saúde no Brasil é indicada em alguns estudos feitos no país (Joshi et al., 2006; Borges et al., 2014; Lacerda et al., 2017; Rabahi et al., 2017).

No estado do Amapá ainda não existem estudos direcionados especificamente para ocorrência de tuberculose em profissionais da saúde. Verifica-se, portanto, a necessidade de um conhecimento sobre a frequência dessa doença nesta população do estado, o que poderá servir de auxílio para novos estudos mais aprofundados sobre este tema e fomentar discussão sobre relação entre profissional da saúde e tuberculose.

O objetivo do presente estudo é quantificar a ocorrência da tuberculose em profissionais da saúde no estado do Amapá entre os anos de 2014 e 2018, a partir do banco de dados de morbidade por tuberculose do SINAN.

\section{Materiais e métodos}

Trata-se de um estudo descritivo de abordagem quantitativa no qual foram utilizados dados secundários sobre casos confirmados de tuberculose em profissionais da saúde entre os anos de 2014 e 2018 no Estado do Amapá obtidos a partir do Sistema de Informação de Agravos de Notificação (SINAN).

As informações coletadas foram transferidas para o programa Tabwin versão 4.1.5, onde se realizaram os cálculos de frequência absoluta e relativa dos números de casos. Os resultados foram transferidos para uma tabela no aplicativo Microsoft Excel 2010, e os resultados, convertidos em tabelas.

Foram incluídos todos os casos notificados de tuberculose em profissionais da saúde diagnosticados com a doença entre os anos de 2014 e 2018 no estado do Amapá. Os casos cuja data de diagnóstico antecede este período foram excluídos. A coleta dos dados foi realizada a partir do banco de dados de morbidade por tuberculose do SINAN, cujo acesso foi feito por meio endereço eletrônico http://tabnet.datasus.gov.br/cgi/tabcgi.exe?sinannet/cnv/tubercap.def

As informações coletadas foram transferidas para o programa Tabwin versão 4.1.5, onde foram realizados os cálculos de frequência absoluta e relativa dos casos notificados. Posteriormente fora criada uma planilha no aplicativo Microsoft Excel 2010 contendo informações com as variáveis município de residência, forma, sexo, raça e faixa etária dos casos notificados e, em seguida, feita a tabulação dos resultados.

A pesquisa se fez de acordo com a Resolução $n^{\circ} 466$ de 2012 da Comissão Nacional de Ética em Pesquisa do Ministério da Saúde, que define as normas éticas regulamentadoras de pesquisas envolvendo seres humanos (Brasil, 2012). Todas as atividades desenvolvidas neste trabalho foram fundamentadas e respaldadas pela Resolução do Conselho Nacional de Saúde.

\section{Resultados e discussão}

Entre 2014 e 2018 foram notificados 28 casos de tuberculose em profissionais da saúde no Estado. A capital, Macapá, registrou o maior número de casos, 23 (82\%). Além de Macapá, registraram casos Calçoene, 2 (7\%), Santana, 2 (7\%), e Porto Grande, 1 (4\%); os demais municípios não apresentaram casos nesse período. A forma predominante da doença foi a pulmonar (90\%). Adicionalmente, observamos na tabela 1 o número de casos confirmados por ano de diagnóstico segundo o município de residência e forma da doença.

O número de casos de tuberculose foi maior no sexo feminino $(67,9 \%)$ do que no masculino $(32,1 \%)$. $68 \%$ dos casos ocorreram em pardos, $18 \%$ em brancos e outros $14 \%$ em negros. Na tabela 2 estão apresentados os casos confirmados por ano diagnóstico segundo sexo e raça.

A tabela 3 apresenta os casos de tuberculose confirmados por ano diagnósticos segundo a faixa etária. $\mathrm{O}$ maior número de casos ocorreu na faixa etária dos 20 a 29 anos (29\%), com apenas um caso de diferença entre a faixa dos 30 a 39 anos (26\%). As faixas com menor ocorrência foram as dos 60 a 69 anos (7\%) e de 80 anos e mais (3\%), respectivamente. 
Tabela 1. Casos confirmados por ano diagnóstico segundo o município de residência e forma.

\begin{tabular}{|c|c|c|c|c|c|c|c|}
\hline Município de residência & 2014 & 2015 & 2016 & 2017 & 2018 & Total & $\%$ \\
\hline Macapá & 2 & 4 & 7 & 1 & 9 & 23 & 82 \\
\hline Calçoene & 0 & 0 & 1 & 1 & 0 & 2 & 7 \\
\hline Santana & 0 & 0 & 1 & 0 & 0 & 1 & 4 \\
\hline Porto Grande & 0 & 1 & 0 & 1 & 0 & 2 & 7 \\
\hline Total & 2 & 5 & 9 & 3 & 9 & 28 & 100 \\
\hline \multicolumn{8}{|l|}{ Forma } \\
\hline Pulmonar & 2 & 5 & 6 & 3 & 9 & 25 & 90 \\
\hline Extrapulmonar & 0 & 0 & 3 & 0 & 0 & 3 & 10 \\
\hline Total & 2 & 5 & 9 & 3 & 9 & 28 & 100 \\
\hline
\end{tabular}

Tabela 2. Casos confirmados por ano diagnóstico segundo sexo e raça.

\begin{tabular}{|c|c|c|c|c|c|c|c|}
\hline Sexo & 2014 & 2015 & 2016 & 2017 & 2018 & Total & $\%$ \\
\hline Feminino & 1 & 4 & 5 & 2 & 7 & 19 & 68 \\
\hline Masculino & 1 & 1 & 4 & 1 & 2 & 9 & 14 \\
\hline Total & 2 & 5 & 9 & 3 & 9 & 28 & 100 \\
\hline \multicolumn{8}{|l|}{ Raça } \\
\hline Branco & 0 & 1 & 2 & 0 & 2 & 5 & 18 \\
\hline Preto & 1 & 1 & 1 & 0 & 2 & 4 & 14 \\
\hline Pardo & 1 & 3 & 6 & 3 & 5 & 19 & 68 \\
\hline Total & 2 & 5 & 9 & 3 & 9 & 28 & 100 \\
\hline
\end{tabular}

Tabela 3. Casos confirmados por ano diagnóstico segundo faixa etária.

\begin{tabular}{lccccccc}
\hline Faixa etária & $\mathbf{2 0 1 4}$ & $\mathbf{2 0 1 5}$ & $\mathbf{2 0 1 6}$ & $\mathbf{2 0 1 7}$ & $\mathbf{2 0 1 8}$ & Total & \% \\
\hline 20 a 29 anos & 1 & 2 & 1 & 0 & 4 & 8 & 29 \\
30 a 39 anos & 1 & 0 & 4 & 0 & 2 & 7 & 26 \\
40 a 49 anos & 0 & 2 & 1 & 1 & 0 & 4 & 14 \\
50 a 59 anos & 0 & 1 & 2 & 2 & 1 & 6 & 21 \\
60 a 69 anos & 0 & 0 & 1 & 0 & 1 & 2 & 7 \\
80 anos e mais & 0 & 0 & 0 & 0 & 1 & 1 & 3 \\
Total & 2 & 5 & 9 & 3 & 9 & 28 & 100 \\
\hline
\end{tabular}

Foi observado no estudo que a maioria dos casos (82\%) ocorreu na capital do estado. A mesma tendência se observa na população geral, que concentra cerca de $70 \%$ dos casos em Macapá. (Brasil, 2018). Isso pode estar relacionado ao fato de a maioria dos serviços de saúde estar concentrada em Macapá e de a cidade conter uma densidade demográfica maior em relação aos outros municípios (IBGE, 2010).

Quanto à forma de apresentação da doença, o resultado desta pesquisa está dentro do que é observado na população geral. Em determinadas condições, a infecção pode evoluir para a doença e se manifestar nas formas pulmonar, extrapulmonar ou mista. A primeira, por ser a mais transmissível, aparece em aproximadamente $80 \%$ dos casos (Veronesi \& Focaccia, 2015). A forma extrapulmonar costuma aparecer em aproximadamente $10-20 \%$ dos casos, mas em indivíduos imunodeprimidos esse percentual pode chegar a $60 \%$ (Bethlem, 2012). Os sintomas da Tb extrapulmonar dependem da região infectada. A pleura é a segunda região mais atingida pela infecção em imunocompetentes e está associada à lesão pulmonar ativa (Lopes, 2006).

Em relação ao sexo, na população geral o número de casos no Brasil é maior em homem do que em mulheres (Viana, 2019). Os resultados da nossa pesquisa diferem desta perspectiva provavelmente devido a maior presença de pessoas do sexo feminino atuando na área da saúde, principalmente nas ocupações em que a infecção e a doença mais ocorre. (Matos et al., 2013). A eficácia das medidas de controle da infecção adotadas pela instituição e a prevalência local são os principais fatores que influenciam na variação do risco de transmissão nosocomial da Tb (Cerqueira et al., 2012; Zhou et al., 2014).

Uma pesquisa realizada em Santa Cruz do Sul - RS com 134 profissionais de saúde da atenção básica revelou uma prevalência de infecção latente por Mycobacterium tuberculosis de 32,8\% nesta população. (Borge et al., 2014). Outro estudo, em Vitória - ES, corrobora a hipótese de que profissionais da saúde da atenção básica estão altamente expostos ao Mycobacterium tuberculosis (Lacerda et al., 2017).

Áreas das unidades onde pacientes com tuberculose pulmonar ou laríngea recebem cuidados e locais onde material biológico potencialmente contaminado com o bacilo é manipulado são considerados de risco. Além disso, surtos nosocomiais estão relacionados com falhas logísticas eu envolvem manejo de pacientes tuberculosos, o isolamento e o reconhecimentos desse paciente (Andrade, 2001; Brasil, 2010). 
Uma limitação da pesquisa foi determinar a ocupação dos casos notificados, já que não há, nos bancos do SINAN, a opção para preenchimento da ocupação na notificação. Seria importante ter conhecimento desta variável para determinarmos qual ocupação foi atingida pela doença. Mas em estudo realizado em um hospital universitário do Espírito Santo, aponta que a maioria dos casos da doença ocorre em profissionais de área da enfermagem. Dos 25 profissionais diagnosticados com tuberculose $32 \%$ eram técnicos em enfermagem e outros $12 \%$ eram enfermeiros; $16 \%$ eram médicos e $8 \%$ técnicos em radiologia, outras categorias somaram, juntas, 32\% dos casos (Prado et al., 2008; Fávero et al., 2012).

E outro estudo realizado também no Espírito Santo revelou uma prevalência de 246/100.000 profissionais em PS da área da enfermagem entre os anos de 2000 a 2008 (Prado et al., 2008). Outra limitação encontrada foi estabelecer um nexo causal entre o local de trabalho do profissional e o local onde ocorreu a infecção do profissional, uma vez que as características de transmissão da doença dificultam saber exatamente onde ocorreu o evento.

Porém, em um estudo realizado na Holanda, no qual se realizou um rastreio microbiológico para identificar as vias de contágio, dos 67 casos de tuberculose registrados em PS, 28 (42\%) adquiriram a infecção no local de trabalho (De Vries, Šebek, \& Weezenbeek, 2006). Um estudo em um hospital de Portugal realizado com 73 profissionais de saúde que teriam entrado em contato com 2 pacientes tuberculosos internados no local demonstrou que, depois da provável exposição, 9 desses profissionais (12,3\%) apresentaram a doença, 8 do sexo feminino e 1 do sexo masculino, e $3(4,1 \%)$ apresentaram infecção latente, o que reforça a vínculo entre exposição e infecção no local de trabalho (Saleiro et al., 2007).

A TB é um risco a que os profissionais de saúde se expõem, especialmente em áreas onde esta doença tem maior prevalência e/ou incidência. O rastreio dos profissionais de saúde com exposição a formas de TB é uma importante ferramenta no diagnóstico precoce de doença ativa, para a identificação dos casos de maior risco de doença futura, ou na identificação de casos da doença nos profissionais de saúde rastreados (Costa et al., 2010; Lacerda et al., 2017). O conhecimento dos dados apresentados reforça a necessidade da adoção de medidas voltadas à detecção, tratamento e acompanhamento de casos.

\section{Conclusão}

A tuberculose se mostra presente na população de profissionais de saúde do estado. Apesar de ser difícil estabelecer um vínculo da infecção com o local de trabalho, é importante reiterar a importância da tomada de medidas de prevenção no ambiente de trabalho dessa população, uma vez que o contato com pacientes bacilíferos ou com material potencialmente contaminado é recorrente. Vale ressaltar aqui que os resultados desta pesquisa apontam também a importância de um fornecimento de dados mais completos pelo SINAN, como, por exemplo, dados sobre a Infecção Latente pelo M. tuberculosis (ILTB) e a ocupação dos casos notificados, o que disponibilizará subsídios mais específicos para futuras pesquisas sobre o tema. Espera-se que este estudo contribua com o conhecimento sobre tuberculose no estado do Amapá e que sirva de fonte de interesse para estudos mais aprofundados sobre a relação entre profissionais de saúde e tuberculose.

\section{Referências}

Andrade, M. K. 2001. Avaliação do Risco de Tuberculose para os Profissionais de Saúde. Boletim de Pneumologia Sanitária, 9(2), 17-20.

Bethlem, E. P. 2012. Manifestações clínicas da tuberculose pleural, ganglionar, geniturinária e do sistema nervoso central. Pulmão RJ, 21(1):19-22.

Borges, T. S., Sonda, E. C., Daronco, A., Battisti, F., Dos Santos, M. M., Valim, A. R., \& Possuelo, L. G. 2014. Prevalência de Infenção Latende por Mycobanteruim tuberculosis em Profissionais de Rede Básica de Saúde. Revista Brasileira em Promoção da Saúde, 27(2), 269-275.

Brasil. 2010. Ministério da saúde. Secretaria de Atenção à Saúde. Hospital Federal de Bonsucesso. Comissão de Controle de Infecção Hospitalar - rotina a13. Rotina para Prevenção de Transmissão de Tuberculose Nosocomial. Disponível em: <https://bit.ly/2XTqTs9>. Acesso em: 05 jun. 2020.

Brasil. 2012. Resolução $n^{\circ} 466$ de 12 de Dezembro de 2012. Define as normas éticas regulamentadoras de pesquisas envolvendo heres humanos. Disponível em: <https://conselho.saude.gov.br/resolucoes/2012/Reso466.pdf>Acesso em: 18 jul. 2019.

Brasil. 2018. Secretaria de Vigilância em Saúde. Boletim epidemiológico. Experiências de Programas de Controle da Tuberculose: 'Porque juntos iremos detectar, tratar e acabar com a tuberculose como problema de saúde pública

Brasil.

Disponível em: 
<http://portalarquivos2.saude.gov.br/images/pdf/2018/outubro/05/Vol.49\%20N\%C2\%BA\%2036.pdf>. Acessado em: 07 jun. 2019.

Brasil. 2019. Ministério da Saúde. Secretaria de Vigilância em Saúde. Boletim epidemiológico. Brasil Livre da Tuberculose: evolução dos cenários epidemiológicos e operacionais da doença. Disponível em: <http://portalarquivos2.saude.gov.br/images/pdf/2019/marco/22/2019-009.pdf>. Acesso em: 04 mai. 2019.

Campos, H. S. 2006. Diagnóstico da tuberculose. Pulmão RJ, 15(2), 92-99.

Cerqueira, A. C., Fávero, J. L., Fregona, G., Maciel, E. L., Prado, T. N., \& Wener, R. C. 2012. Prevalência de Tuberculose em Profissionais da Área de Enfermagem Obtida por Método de Relacionamento de Bancos de Dados, 2000 a 2008, Espírito Santo. Revista Brasileira de Pesquisa em Saúde, 14(2), 31-37.

Costa, J. T., Silva, R., Sá, R., Cardoso, M. J., Ferreira, J., Ribeiro, C., Miranda, M., \& Plácido, J. L. 2010. Tuberculose - Risco de transmissão continuada em profissionais de saúde. Revista Portuguesa de Pneumologia, 16(1), 5-21.

De Vries, G., Šebek, M., \& Weezenbeek, C. 2006. Healthcaren workers with tuberculosis infected during work. European Respiratory Journal, 8(6), 1216-1221.

Fávero, J. L., Cerqueira, A. C. B., Fregona, G., Prado, T. N., Werner, R. C. D., \& Maciel, E. L. N. 2012. Prevalência de Tuberculose em Profissionais da Área de Enfermagem Obtida por Método de Relacionamento de Bancos de Dados, 2000 a 2008, Espírito Santo. Revista Brasileira de Pesquisa em Saúde, 14(2), 31-37.

Instituto Brasileiro de Geografia e Estatística (IBGE). 2010. Estatísticas da Saúde: Assistência Médica Sanitária 2009. Rio de Janeiro, RJ: IBGE.

Joshi, R., Reingold, A. L., Menzies, D., \& Pai, M. 2006. Tuberculosis Among Health-Care Workers in Low and Midle-Income Countries: A Systematic Review. Plos Medicine, 3 (12), e494.

Lacerda, T. C., Souza, F. M., Prado, T. N., Locatelli, R. L., Fregona, G., Lima, R. D., \& Maciel, E. L. 2017. Infecção por Tuberculose entre Profissionais da Sáude de Atenção Básica. Jornal Brasileiro de Pneumoloigia, 43(5), 416-423.

Lopes, A. J. 2006. Tuberculose Extrapulmonar: aspectos clínicos e de imagem. Pulmão RJ, 15(4), 253-261.

Matos, I. B., Ceriotti, T., Ramona, F., \& Oliveira, M.C. 2013. Profissões e ocupações de saúde e o processo feminização: tendências e implicações. Athenea Digital, 13(2), 239-244.

Prado, T. N., Galavote, H. S., Brioshi, A. P., Lacerda, T., Fregona, G., Detoni, V. V., ... Maciel, E. L. N. 2008. Perfil epidemiológico dos casos notificados de tuberculose entre os profissionais de saúde no Hospital Universitário em Vitória (ES) Brasil. Jornal Brasileiro de Pneumologia, 34(8), 607-613

Rabahi, M. F., Júnior, J. L., Ferreira, A. C., Tannus-Silva, D. G., \& Conde, M. B. (2017). Tratamento da Tuberculose. Jornal Brasileiro de Pneumoloia, 5(43), 472-486

Saleiro, S., Santos, A. R., Vidal, O., Carvalho, T., Costa, J. T., \& Marques, J. A. 2007. Tuberculose em Profissionais de Saúde de um Serviço Hospitalar. Revista Portuguesa de Pneumologia, 13(6), 789-799.

Veronesi, R., \& Focaccia, R. 2015. Tratado de Infectologia (5a ed.). São Paulo, SP: Atheneu.

Viana, P. V. S. 2014. Tuberculose no Brasil: uma análise dos dados de notificação, segundo macro-região e raça/cor, para o período 2008-2011. Dissertação de mestrado (Escola Nacional de Saúde Pública Sergio Arouca), Rio de Janeiro, RJ: Fiocruz.

World Health Organization [WHO]. 2018. Global Tuberculosis Report 2018. Disponível em: $<$ http//www.who.int/tb/publications/global_report/tb18_ExecSum_web_4Oct18.pdf? ua=1>. Acesso em: 21 set. 2019.

Zhou, F., Zhang, L., Gao, L., Hao, Y., Zhao, X., Liu, J., Lu, J., Li, X., Yang, Y., Chen, J., \& Deng, Y. 2014. Latent Tuberculosis Infection and Occupational Protection among Health Care Workers in Two Types of Public Hospitals in China. PLoS One, 9(8), e104673.

\section{Minicurrículo}

Luciane de Almeida Ferreira. Bacharelado em Biomedicina da Faculdade Estácio de Macapá. Macapá, Amapá, Brasil.

Myllon Ramirio Silva da Luz. Bacharelado em Biomedicina da Faculdade Estácio de Macapá. Macapá, Amapá, Brasil. 
Claude Porcy. Graduação em Biomedicina, Especialização em Análises Clínicas; e em Tecnologia de Software, mestrado em Biologia Parasitária (área de concentração em Biologia da Interação Patógeno-Hospedeiro). Atualmente é Professor de Microbiologia e Bacteriologia da Faculdade Estácio de Macapá e Biomédico do Governo do Estado do Amapá. Tem experiência em Análises Clínicas com ênfase nas áreas da Hematologia e Microbiologia médica.

Flavio Henrique Ferreira Barbosa. Professor Adjunto II (Dedicação Exclusiva) da Universidade Federal de Sergipe - UFS, vinculado ao Departamento de Morfologia - DMO na Área de Conhecimento: Microbiologia e Imunologia. Possui Graduação em Ciências Biológicas pelo Centro Universitário Metodista Izabela Hendrix/MG (2003), Especialista em Saúde Pública (em andamento) pela Fundação Estadual de Saúde de Sergipe - FUNESA/Universidade Federal de Sergipe - UFS (2020), Especialista em Gestão e Manejo Ambiental pela Universidade Federal de Lavras - UFLA/MG (2008), Especialista em Didática e Metodologia do Ensino Superior pela Anhanguera Educacional/DF (2009), Mestrado em Microbiologia pela Universidade Federal de Minas Gerais - UFMG (2006) e Doutorado em Microbiologia pela Universidade Federal de Minas Gerais - UFMG (2010). Realizou estágio Pós-Doutoral na Universidade Rovira i Virgili, Espanha (2018-2019) na área de Fermentação e Tecnologia Cervejeira (Cerveja Artesanal). Formou-se Mestre Cervejeiro Artesanal e Sommelier (2019). Tem como linhas de pesquisa estudos de microbiologia das fermentações; leveduras de cervejaria e tecnologia cervejeira. Já atuou em pesquisas nas linhas de ecologia e fisiologia microbiana das superfícies e mucosas do hospedeiro humano e outros animais; estudo e desenvolvimento de probióticos e prebióticos; biotecnologia e biodiversidade de microrganismos; e pesquisa de substâncias antagonistas e antimicrobianas produzidas por microrganismos e plantas. É líder do Grupo de Pesquisa: Microbiologia das Fermentações e Tecnologia Cervejeira (www.cervejaufs.com.br). É integrante da equipe de pesquisadores do Grupo de Pesquisa: Microbiologia e Imunologia (UFS) e Microbiologia Aplicada (UFS).

Rubens Alex de Oliveira Menezes. Graduação em Enfermagem (Bacharelado e Licenciatura) pela Universidade Federal do Amapá (2009). Pós-Graduação em Microbiologia Nível de Especialização pela Universidade de Fortaleza, Pós-Graduação em Docência do Ensino Superior pela Faculdade de Tecnologia do Amapá - META, Pós-graduação em Gestão em saúde pública pela Faculdade de Macapá - FAMA, PósGraduação Nível de Mestrado em Ciências da Saúde Área de Concentração: Epidemiologia e Saúde Pública pela Universidade Federal do Amapá (2013). Pós-graduação Nível de Doutorado em Biologia de Agentes Infecciosos e Parasitários (PPG BAIP) pela Universidade Federal do Pará. Atualmente é docente do curso de enfermagem da Universidade Federal do Amapá (UNIFAP), Adjunto I, atuando na linha de pesquisa direcionada as doenças emergentes, reemergentes e negligenciadas, desenvolvendo estudos com enfoque especial em: Saúde pública e epidemiologia e controle de doenças transmissíveis, não transmissíveis, infecciosas e parasitárias de interesse médico-sanitário.

Como citar: Ferreira, L.A., Luz, M.R.S., Porcy, C., Barbosa, F.H.F., \& Menezes, R.A.O. 2020. Ocorrência da tuberculose em profissionais da Saúde entre os anos 2014 e 2018 no Amapá. Pubsaúde, 3, a037. DOI: https://dx.doi.org/10.31533/pubsaude3.a037

Recebido: 21 mai. 2020.

Revisado e aceito: 27 mai. 2020.

Conflito de interesse: os autores declaram, em relação aos produtos e companhias descritos nesse artigo, não ter interesses associativos, comerciais, de propriedade ou financeiros que representem conflito de interesse.

Licenciamento: Este artigo é publicado na modalidade Acesso Aberto sob a licença Creative Commons Atribuição 4.0 (CC-BY 4.0). 